\title{
BMJ Open Health system costs of potentially inappropriate prescribing in Ontario, Canada: a protocol for a population- based cohort study
}

\author{
Cody D Black, ${ }^{1}$ Kednapa Thavorn, ${ }^{1,2,3}$ Douglas Coyle, ${ }^{1}$ Glenys Smith, ${ }^{3}$
} Lise M Bjerre ${ }^{1,3,4,5}$

To cite: Black CD, Thavorn $\mathrm{K}$, Coyle D, et al. Health system costs of potentially inappropriate prescribing in Ontario, Canada: a protocol for a populationbased cohort study. BMJ Open 2018;8:e21727. doi:10.1136/ bmjopen-2018-021727

- Prepublication history and additional material for this paper are available online. To view these files, please visit the journal online (http://dx.doi. org/10.1136/bmjopen-2018021727).

Received 15 January 2018 Revised 14 March 2018 Accepted 18 April 2018

\section{Check for updates}

${ }^{1}$ School of Epidemiology and Public Health, University of Ottawa, Ottawa, Ontario, Canada ${ }^{2}$ Ottawa Hospital Research Institute, The Ottawa Hospital, Ottawa, Ontario, Canada ${ }^{3}$ ICES uOttawa, Institute for Clinical Evaluative Sciences, Ottawa, Ontario, Canada ${ }^{4}$ Department of Family Medicine, University of Ottawa, Ottawa, Ontario, Canada

${ }^{5}$ Bruyère Research Institute, Ottawa, Ontario, Canada

Correspondence to Dr Lise M Bjerre; Ibjerre@bruyere.org

\section{ABSTRACT}

Introduction Adverse drug events (ADEs) are common in older persons and contribute significantly to emergency department visits, hospitalisations and mortality. ADEs are often due to potentially inappropriate prescriptions (PIP) or potentially inappropriate omissions (PIO), and are avoidable if inappropriate prescriptions or omissions are identified and prevented. Identifying PIP/PI0 at the population level through the application of PIP/PI0 assessment tools to health administrative data can provide a unique opportunity to assess the economic burden of PIP/PIO on the healthcare system beyond medication costs which is yet to be done. The objective of this study is to assess the economic burden associated with PIP/PIO and to estimate the incremental costs associated with distinct PIP/PIO in the province of Ontario.

Methods and analysis We will conduct a retrospective cohort study using Ontario's health administrative databases. Eligible patients aged 66 years and older who were prescribed at least one medication between 1 April 2003 and 31 March 2014 (approximately 2.4 million patients) will be included. Population attributable fraction methodology will be used to assess the overall burden of PIP in Ontario, while regression analyses will be used to estimate the incremental costs of having specific PIP criteria and aid in prioritising targets for intervention. Ethics and dissemination This study was approved by the Institutional Review Board at Sunnybrook Health Sciences Centre, Toronto, Canada. Dissemination will occur via publication, presentation at national and international conferences, and knowledge exchange with various stakeholders.

\section{INTRODUCTION \\ Background}

Adverse drug events (ADEs) are common in older persons due to physiological vulnerability associated with ageing and disease. ${ }^{1}$ These contribute significantly to emergency department (ED) visits, unplanned hospitalisations, ${ }^{2}$ and in-hospital morbidity and mortality. ${ }^{3}$ Many of these ADEs are due to potentially inappropriate prescriptions (PIPs) or potentially inappropriate omissions (PIOs)

\section{Strengths and limitations of this study}

- This unique study provides an analytical framework to estimate the economic impact of potentially inappropriate prescribing (PIP) at the population level.

- This study benefits from being able to incorporate costs of hospitalisation and emergency department visits in addition to medication costs in the assessment of PIP-related costs, as well as the ability to adjust for potential confounders beyond age and sex, providing a less biased estimate of costs associated with PIP.

- This study has limitations common to studies using health administrative data, primarily uncertainty surrounding patient adherence to medication and lack of documentation of some potential confounders.

- Identifying the overall costs associated with PIP would provide further evidence to support PIP as a high priority for healthcare decision-makers, while characterising the costs of specific PIP scenarios would provide these decision-makers with actionable targets to address PIP.

(alternatively named potential prescribing omissions) and are avoidable if inappropriate prescriptions and/or omissions are identified and prevented. PIP/PIO is also most common in older persons and the likelihood of PIP increases as patients are prescribed more drugs than may be clinically necessary, which is defined as polypharmacy. ${ }^{4}$

A number of tools have been developed to identify PIP/PIO (referred to as PIP from now on unless otherwise specified) in clinical settings, including the STOPP/START criteria $^{5}$ (Screening Tool of Older Persons' Prescriptions/Screening Tool to Alert doctors to Right Treatment) and the Beers criteria. ${ }^{6-10}$ These tools are time consuming and costly to apply individually and are underused. Applying these tools to assess the appropriateness of prescribing to population-wide health administrative data can provide a 


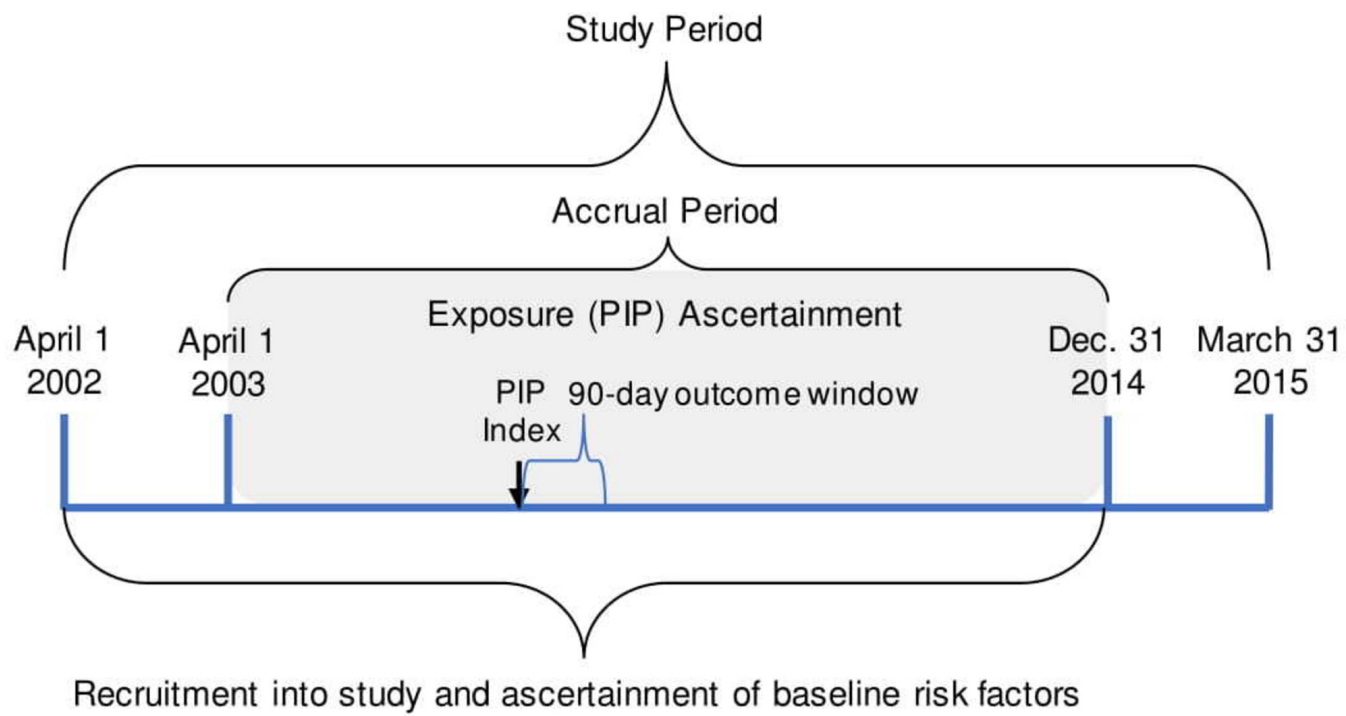

Figure 1 Definition of observation period. PIP, potentially inappropriate prescription.

unique opportunity to assess both the frequency of PIP and its associated costs, in terms of medication and health services use, at both the individual and population level.

\section{Evidence gaps to be filled}

PIP is associated with increased costs and health services use according to studies conducted in Ireland and the $\mathrm{UK},{ }^{11-13}$ but its economic impact in Canada is unknown. The PIP-STOPP study is a CIHR-funded project with the aim of using subsets of the STOPP/START and Beers' criteria applicable to health administrative data housed at the Institute for Clinical Evaluative Sciences (ICES) to identify PIP and PIO at the population level in Ontario, its association with hospitalisations, ED visits and mortality, and health system costs. ${ }^{14}$

Identifying the overall health system costs associated with PIP would provide further evidence to support PIP as a high priority for decision-makers by quantifying its overall health system burden, while characterising the costs of specific PIP scenarios would provide these decision-makers with actionable targets to address PIP through a better understanding of the flow of costs attributable to different PIP scenarios and the identification of higher cost-burden PIPs.

This study protocol details the methods to assess the health system costs associated with PIP overall in Ontario, as well as to estimate the incremental costs of PIP scenarios which vary by their downstream healthcare resource use and by frequency. To address these knowledge gaps, the objectives of this study are to:

- Assess the overall health system costs associated with PIP in Ontario.

- Estimate the incremental costs associated with distinct PIP scenarios occurring in Ontario.

\section{METHODS}

Methods regarding the study design, participants, datasets, exposure and cohort size for this study have been extensively described previously in the published protocol of its parent study ${ }^{14}$ and are only summarised here. The methods common to both objectives, including study design, participants and data sources, are listed first, followed by the exposure, outcomes, covariates and statistical analyses relevant to each objective.

\section{Study design}

A population-based, retrospective cohort study design will be used, identical to that from previous publications from a larger retrospective, population-based cohort study on the identification of PIP and predicting patient outcomes in Ontario. ${ }^{14}$

\section{Definition of observation periods}

The study period will span from 1 April 2002 to 31 March 2015 , which is based on the availability of the necessary databases. The patient accrual period will be from 1 April 2003 to 31 December 2014, allowing for a 1-year lookback period for prior health services utilisation and baseline covariates, as well as a 90-day follow-up period after the last possible PIP to allow time for identification of associated outcomes, figure 1. We have chosen a 90-day observation window following instances of PIP in which to identify outcomes because we do not assume that the potential influence of PIP would extend beyond this period.

\section{Participants}

From the data housed at ICES, a large cohort of patients who are eligible for the study (approximately 2 million participants) has been identified. These patients are Ontario residents aged 66 years and older with Ontario Health Insurance Plan (OHIP) drug coverage (approximately $97 \%$ of Ontario seniors) who have been dispensed at least one prescription between 1 April 2003 and 31 December 2014.

\section{Data sources}

The proposed project will use population-level health administrative data from Ontario, which is housed at ICES. ${ }^{1516}$ Accessing data through ICES allows for the linking 
of demographic, socioeconomic, hospital and outpatient health services, physician billing datasets, and prescription dispensation to Ontarians aged 65 years or older, or those requiring social assistance. ${ }^{17}$ Cost data are available for all physician claims, hospitalisations, ambulatory care services, home care services, long-term and complex continuing care, and medical devices in Ontario, while prescription medication cost data are available for those 65 years of age or older and those requiring social assistance. The following datasets will be linked to gather the appropriate and available exposure, outcome and covariate data necessary for analyses: Ontario Drug Benefits Claims Database (ODBD), Discharge Abstract Database, Same Day Surgery Database, National Ambulatory Care Reporting System, OHIP database, Registered Persons Database (see online supplementary appendix A for brief description of each). Additionally, five ICES-derived cohorts will be used for case ascertainment for specific STOPP/START criteria requiring the following diagnoses: asthma, diabetes, hypertension, chronic obstructive pulmonary disease and congestive heart failure. ${ }^{18}$

\section{Objective 1: overall health system costs due to PIP Exposure}

The subset of STOPP/START criteria applicable to health administrative databases were identified based on the availability of the data necessary for their use and coded into a format applicable to ICES-housed health administrative data using a combination of medication, diagnostic (ie, International Classification of Diseases Codes), healthcare services utilisation and physician billing codes. This process identified $64 \%$ of STOPP criteria and $27 \%$ of START criteria as applicable to health administrative data available through ICES. A manuscript describing the coding process in detail is in preparation. ${ }^{19}$

The exposure of interest will be the first occurrence of any PIP identified using health administrative databases for each patient in the cohort during the study accrual period from 1 April 2003 to 31 December 2014. Unexposed patients will be those who have not experienced any PIP during the study accrual period.

\section{Outcome}

The primary outcome will be the combined medication, hospitalisation and ED visit costs attributable to PIP paid by the Ontario Ministry of Health and Long-Term Care over the 3 months following a PIP. These costs, from documented clinical events attributable to PIP, will be identified via population attributable fraction (PAF) methods. ${ }^{2021}$ To identify costs, the ICES costing algorithm will be used.$^{22}$ This algorithm allows for the identification of costs from any health services covered by the OHIP over a defined time period. ${ }^{22}$ All costs will be inflated to 2015 \$C using Statistic Canada's Consumer Price Index. ${ }^{23}$ The PAF estimates for hospitalisations, ED visits and medications will be derived via the incidence rate ratios (IRRs) obtained from Poisson or negative binomial regression models for each outcome, described in further detail in the statistical analyses section. Each PAF estimate will then be multiplied by the total cost of their respective healthcare service over the 90-day observation period for the whole population, which will be totalled to obtain the total costs attributable to PIP over the study period.

Though we realise not all hospitalisations and ED visits will be causally related with PIP, ADEs are frequently under-recognised during ED visits and hospitalisation. ${ }^{24}{ }^{25}$ As such, we chose to use reliably documented clinical events (hospitalisation and ED visits) and their costs, along with medication costs, as most suitable for the primary outcome. These costs were selected as they are the costs associated with the most reliably documented clinical events and those most likely to be linked to PIP. For medication costs, only those attributable to STOPP criteria will be determined and patients with PIP who either have a START criterion as their first PIP, or multiple first PIPs, will be excluded from this analysis. This is to ensure that all drug costs are attributable to the prescribing of a medication (ie, STOPP criteria) and not to the omission of medication (ie, START criteria), which would in fact result in a reduction in costs.

\section{Covariates}

Analyses will be adjusted for potential confounders which are available through provincial health administrative databases and either known or perceived to be associated with PIP or with our outcomes of interest (ie, hospitalisation, ED visits and medication use), including age, ${ }^{2627}$ sex, ${ }^{27}$ income quintile, ${ }^{27}{ }^{28}$ rurality, aggregated diagnosis group score (ie, comorbidity status), 272930 number of unique drug identification numbers (ie, pills prescribed concurrently) in year prior to PIP, ${ }^{31-35}$ number of prescribers in year prior to PIP, ${ }^{36}$ whether the patient had a MedsCheck (billable medication review performed by a pharmacist, usually in the community) in year prior to PIP, number of days spent in hospital in year prior to PIP, ED visit in 6 months prior to PIP. ${ }^{26} 272937-41$

For this objective, the ascertainment of covariate status will be done at the PIP date for participants experiencing a PIP and at the randomly assigned index date for participants who do not experience a PIP, as further described below.

\section{Statistical analyses}

The direct costs associated with the occurrence of any first PIP in Ontario will be estimated by combing medication-related and non-medication-related costs attributable to PIP via the steps below.

\section{Assignment of time to PIP for unexposed patients}

To estimate the health system costs due to PIP, it is necessary to compare the number of days spent in hospital, the number of ED visits and the volume of newly prescribed medications in participants with and without PIP. To do this, we will need to assign an index date to participants without PIP so that they have comparable lookback and observation windows for covariate and outcome ascertainment, respectively. The index date for participants not 
experiencing a PIP will be treated as missing at random. We will conduct parametric survival analysis on the participants within the cohort who have experienced a PIP, with their time-to-first PIP from cohort entry as the response variable in the model. The models will be stratified by sex and median age during cohort participation according to the following categories: $66-70,71-75,76-80,81-85$, $86-90$, over 90 . The models will be fitted to identify the appropriate distribution for its random error component. Once the best-fitting model has been identified, the resulting distribution and appropriate shape and scale parameters, depending on the distribution identified, will be applied to the participants without a PIP via the random number function in $\mathrm{SAS}^{42}$ in order to randomly assign them a PIP index date and identify the start of their 90-day observation window. This random assignment will be done three times, with the first randomly assigned PIP index used for the primary analyses and most other subgroup and sensitivity analyses, while the other two will be used to conduct a sensitivity analysis to determine whether the random assignment method used impacts study results. Participants with an assigned PIP index date that falls beyond the end of their follow-up period will be excluded from all analyses. For both exposed and unexposed participants, we will determine whether they have at least 90 days between their PIP index date and the end of their follow-up. If this difference is less than 90 days, participants will be removed from the analysis.

This approach to assignment of an index date in non-PIP participants was selected over simple random assignment of time to PIP since the underlying distribution of time-to-first PIP in participants experiencing PIP is unknown and is not believed to be normally distributed and is also thought to vary by age and sex. We do not consider matching as an option due to the high prevalence of PIP in the older population resulting in a difficulty to find suitable matches for all participants with a PIP. Additionally, the first day of a participant's inclusion in the study population for participants without PIP cannot be used as their PIP index date as this would make the unexposed group systematically younger than those exposed to PIP during analyses, resulting in confounding that may not be completely adjusted for even though age is being included in analyses as a covariate.

\section{Medication use, ED visits and hospitalisations attributable to PIP}

The IRRs for ED visits, days spent in hospital and medications for participants with PIP versus those without PIP will be determined via parameter estimates for the PIP variable from regression models for count data. Three models will be created, each with the response variable of either count of ED visits, days spent in hospital and total number of unique, newly prescribed medications in the 90-day period following their PIP date for participants with PIP, or the randomly assigned PIP index date for participants not experiencing a PIP. Each of the models will be adjusted for the covariates listed previously. For each outcome, either a Poisson or negative binomial model will be fitted based on a comparison of the mean of the response variable with its variance to determine if overdispersion is present. ${ }^{43}$ Should the mean and variance be equal, or approximately so, then the Poisson model will be used. If the variance is greater and proportional to the mean, indicating overdispersion, then the negative binomial model will be used. The IRRs obtained via the parameter estimate for the PIP variable in the best-fitting model for each outcome will be used to derive their respective PAF in combination with the proportion of the population exposed to PIP $\left(\mathrm{P}_{\text {exp }}\right)$, obtained directly from our cohort as the PIP prevalence in the whole study population, via Levin's formula: $\left(\left(\mathrm{P}_{\exp } *(\mathrm{RR}-1)\right) /\left(\left(\mathrm{P}_{\exp }(\mathrm{RR}-\right.\right.\right.$ 1))+1))*100. ${ }^{2021}$

\section{Total costs attributable to PIP}

The respective PAFs for ED visits, days spent in hospital and medications will be multiplied by the total costs for each of these health services, respectively, to obtain the total cost of each service use attributable to PIP over the 90-day observation period. These costs will then be combined to obtain the total health system costs attributable to PIP over the study period. The same methods will be used to obtain costs attributable to PIP by year.

\section{Subgroup analyses}

Similar methods to those listed above will be conducted in order to obtain the costs attributable to PIP by age, categorised by the median age of cohort participation based on the categories described above, and sex. Additionally, a subgroup analysis for the ED visit and hospitalisation costs will be conducted based on whether a patient's first PIP was a STOPP or START criteria, with patients experiencing multiple first PIPs excluded.

\section{Objective 2: incremental costs of specific PIP criteria Exposure}

PIP will be identified using the same methods described in objective 1. Specific PIP criteria will be selected for the assessment of the incremental costs associated with each of them. For each of the criteria studied, the exposure of interest will be the first time the PIP of interest occurred for each participant in the cohort. Exposed participants must have never experienced another PIP prior to the PIP date, as well as during the 90-day observation window. Unexposed participants will not have experienced any PIP during their cohort participation.

To identify the PIPs to model, each of the individual PIPs applicable to health administrative data was plotted on a graph according to their frequency (y-axis) by their crude average healthcare services costs (ie, medication, hospitalisation and ED visits combined (x-axis), figure 2. Two STOPP criteria falling on the high-cost plane were identified due to their potential for being high-impact PIPs, along with a high-frequency START criterion which also fell near this plane. A low-frequency/low-cost STOPP criteria was chosen to be used for comparison. The PIPs identified were: START A6, STOPP J6, STOPP K2, STOPP 


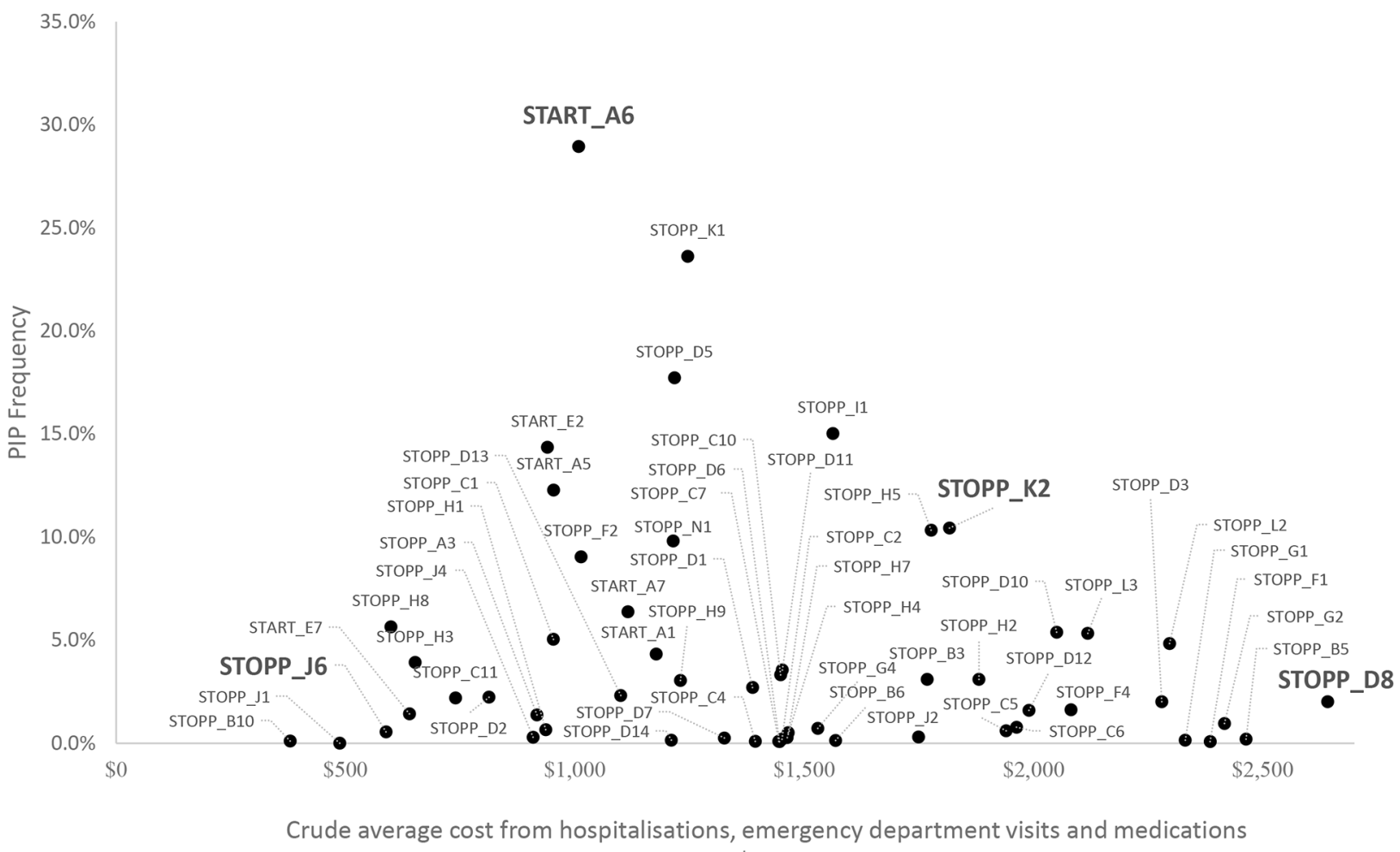

(\$CAD)

Figure 2 Assessment of potentially inappropriate prescription (PIP) frequency by crude costs (from hospitalisations, emergency department visits and medications) to determine PIP scenarios to be modelled.

D8. START A6 is defined as the omission of ACE inhibitor with systolic heart failure and/or documented coronary artery disease. STOPP K2 is defined as the use of any neuroleptic drug. STOPP D8 is flagged as a PIP when anticholinergics and or antimuscarinics are prescribed in participants with delirium or dementia, due to risk of exacerbation of cognitive impairment. The final selected PIP, STOPP J6, is defined as the prescribing of androgens (male sex hormones) in the absence of primary or secondary hypogonadism. The full definitions as they appear in the STOPP/START criteria for each of the selected criteria are described in table 1, along with their categorised frequency and crude costs. ${ }^{5}$

\section{Outcome}

The outcome will be the combined hospitalisation, ED visit and PIP medication costs in the 90-day period following the PIP date. The ED visit and hospitalisation costs will be obtained via the ICES costing algorithm ${ }^{22}$ and inflated to 2015 \$C. Like the hospitalisation and ED visit costs, we will attempt to obtain the PIP medication cost via the ICES costing algorithm, though it may not be possible to obtain only the medication cost for the PIP medication, as the macro currently obtains all medication costs over a designated period as opposed to the costs of each individual medication. Should we be unable to obtain PIP medication costs via the macro, we will identify the lowest hypothetical medication costs for each PIP via the prescribe smart mobile application, ${ }^{44}$ which provides the drug unit price of all available drugs within
Ontario and allows for easy comparison within class, at WHO Defined Daily Dose for each drug. ${ }^{45}$ The drug cost of a daily dose will be multiplied by 90 to obtain the most conservative estimate of a 90-day supply to match the observation window. The highest and median prices for each PIP will also be obtained and used in sensitivity

Table 1 Definitions for PIP criteria to be modelled as part of the second objective, along with their frequency and cost classification

\begin{tabular}{|c|c|c|c|}
\hline PIP & Definition & $\begin{array}{l}\text { PIP } \\
\text { frequency }\end{array}$ & Costs \\
\hline $\begin{array}{l}\text { START } \\
\text { A6 }\end{array}$ & $\begin{array}{l}\text { ACE inhibitor with systolic heart } \\
\text { failure and/or documented } \\
\text { coronary artery disease. }\end{array}$ & High & Mid \\
\hline $\begin{array}{l}\text { STOPP } \\
\text { K2 }\end{array}$ & Neuroleptic drugs. & Mid & Mid \\
\hline $\begin{array}{l}\text { STOPP } \\
\text { D8 }\end{array}$ & $\begin{array}{l}\text { Anticholinergics/antimuscarinics in } \\
\text { patients with delirium or dementia } \\
\text { (risk of exacerbation of cognitive } \\
\text { impairment). }\end{array}$ & Low & High \\
\hline $\begin{array}{l}\text { STOPP } \\
\text { J6 }\end{array}$ & $\begin{array}{l}\text { Androgens (male sex hormones) } \\
\text { in the absence of primary or } \\
\text { secondary hypogonadism } \\
\text { (risk of androgen toxicity; no } \\
\text { proven benefit outside of the } \\
\text { hypogonadism indication). }\end{array}$ & Low & Low \\
\hline
\end{tabular}

PIP, potentially inappropriate prescription; STOPP, Screening Tool of Older Persons' Prescriptions; START, Screening Tool to Alert doctors to Right Treatment. 
analyses, should this approach be used, to observe the PIP costing method's impact on incremental costs.

\section{Covariates}

The covariates of interest for objective 2 will be the same as in objective 1 . Where they differ is in the assignment of the PIP date for ascertainment of covariate status in participants not experiencing a PIP. In objective 2, a subset of participants not experiencing a PIP will be selected for inclusion into the analysis based on having an exact, or similar, index date as a participant with a PIP. Once included, participants without a PIP will be assigned the same PIP date as the person with whom they share the exact or similar index date. This approach to participant inclusion and PIP date assignment has been selected since an efficient approach for participant selection and PIP date assignment that would be least likely to bias study results is necessary. The number of participants with the PIPs of interest ranges from several thousand to over 200000 , thus the number of unexposed participant's needs to be reduced in size for each of the analyses, precluding the inclusion of all unexposed participants in the cohort. Additionally, the index date is the least likely of all available covariates at time of participant selection to be associated with PIP or cost and outcomes, thus reducing the potential of introducing bias in participant selection.

\section{Statistical analyses}

The incremental costs associated with having each of the individual PIP criterion described in table 1 versus not having the PIP in question will be modelled using regression analyses. Due to the typical distribution associated with cost data, multiple candidate models will be assessed to identify the model that best fits the data. This process will begin with the fitting of an ordinary least squares regression and assessment of model fit, as well as a check for heteroscedasticity. If there is evidence of heteroscedasticity, we will then proceed with the selection of a generalised linear model. The link function and variance structure to be used will be determined using the TRANSREG function in SAS and modified Park's test, respectively. Should the Poisson variance structure be selected, the mean of the costs will be compared with the variance to determine whether overdispersion is present and whether a negative binomial model is preferred. All candidate models will be adjusted for the covariates described above. Each model's performance will be assessed using the Bayesian information criterion and graphical check of the distribution fit comparing the distribution of the predicted values with the expected values.

\section{Patient and public involvement}

The research questions addressed by this protocol were informed by the values and preferences of patients with regards to reducing their medication burden and improving their health outcomes, though no patients or public were directly involved in the development of this protocol. Results of this study will be disseminated to patient groups within our research networks.

\section{ETHICS AND DISSEMINATION}

\section{Safety and confidentiality considerations}

This study makes use of previously collected health administrative databases housed at ICES, accessed from ICES@uOttawa, and does not require any additional intervention or data collection at the patient level. ICES links deidentified population-based health information at the population level in a way that ensures privacy and confidentiality to participants. As per ICES procedures, cell sizes of less than five will not be reported to address concerns about possible breaches of confidentiality.

\section{Dissemination}

Dissemination will primarily occur via publication of study results and presentation at national and international conferences. Professional networks will be used to promote dissemination with various stakeholders, including health policy-makers at the provincial and national levels.

\section{Statement of originality}

The assessment of the economic burden of PIP and PIO at the population level using linked health administrative databases is the first of its kind in any jurisdiction to the best of our knowledge. This is the first study of its kind leveraging linked health administrative databases to assess the health services costs of PIP and PIO beyond the medication costs alone, as well as the first study that will be able to adjust for potential confounders beyond age and sex.

\section{Anticipated limitations}

Our study is subject to limitations that are common to studies relying on health administrative databases which may affect our estimates of the prevalence of PIP and associated costs. It cannot be confirmed that participants adhered to the instructions regarding the medications dispensed to them, or regularly took them. Adherence to medication can only be assessed by comparing the date when an original prescription was scheduled to expire with the dispensation date of the renewal prescription. It is also difficult to determine whether some of the PIP identified by the STOPP/START criteria are indeed inappropriate without clinical or diagnostic data that are unavailable to us. ${ }^{46}$ Some of the STOPP/START criteria include over-the-counter medications or medications that are not covered by Ontario's public medication plan $(\mathrm{ODB})$, and thus are not captured in the ODBD or identified as a PIP in our database. Additionally, we are unable to determine the cost of PIP medications from the linked health administrative databases due to the complexity and time required to identify specific prescriptions from a total of over half a billion prescriptions. As such, a 
conservative approach will be used to identify medication costs as described in the outcomes section. Despite these limitations, we are confident our study will produce useful, conservative estimates of the health system costs associated with PIP.

Contributors LMB wrote the original grant application for this project and conceived the initial idea. CDB led the development of this subsequent protocol. The final methodology listed in this protocol was developed collaboratively by $\mathrm{CDB}, \mathrm{LMB}, \mathrm{KT}, \mathrm{DC}$ and GS, with LMB, KT, DC and GS providing expertise in their respective fields. Additionally, CDB, LMB, KT, DC and GS reviewed the manuscript for important intellectual content, made suggestions for improvement as appropriate and approved the final version.

Funding This work was supported by the Canadian Institutes of Health Research (CIHR), grant number 287245-HPM-BRUY-46830 (principal investigator: LMB). CDB is supported by a ClHR Drug Safety and Effectiveness Cross-disciplinary Training program traineeship and a University of Ottawa Excellence Scholarship.

Competing interests None declared.

Patient consent Not required.

Ethics approval This study was approved by the institutional review board at Sunnybrook Health Sciences Centre, Toronto, Canada.

Provenance and peer review Not commissioned; externally peer reviewed.

Open access This is an open access article distributed in accordance with the Creative Commons Attribution Non Commercial (CC BY-NC 4.0) license, which permits others to distribute, remix, adapt, build upon this work non-commercially, and license their derivative works on different terms, provided the original work is properly cited and the use is non-commercial. See: http://creativecommons.org/ licenses/by-nc/4.0/

(C) Article author(s) (or their employer(s) unless otherwise stated in the text of the article) 2018. All rights reserved. No commercial use is permitted unless otherwise expressly granted.

\section{REFERENCES}

1. Spinewine A, Schmader KE, Barber N, et al. Appropriate prescribing in elderly people: how well can it be measured and optimised? Lancet 2007;370:173-84.

2. Laroche ML, Charmes JP, Nouaille Y, et al. Is inappropriate medication use a major cause of adverse drug reactions in the elderly? Br J Clin Pharmacol 2007;63:177-86.

3. Lazarou J, Pomeranz BH, Corey PN. Incidence of adverse drug reactions in hospitalized patients: a meta-analysis of prospective studies. JAMA 1998;279(15):1200-5.

4. Fulton MM, Riley Allen E, Allen E. Polypharmacy in the elderly: A literature review. J Am Acad Nurse Pract 2005;17:123-32.

5. O'Mahony D, O'Sullivan D, Byrne S, et al. STOPP/START criteria for potentially inappropriate prescribing in older people: version 2. Age Ageing 2015;44:213-8.

6. Beers $\mathrm{MH}$, et al. Explicit Criteria for Determining Inappropriate Medication Use in Nursing Home Residents. Arch Intern Med 1991;151:1825.

7. Beers MH. Explicit criteria for determining potentially inappropriate medication use by the elderly. An update. Arch Intern Med 1997;157:1531-6.

8. Fick DM, Cooper JW, Wade WE, et al. Updating the Beers criteria for potentially inappropriate medication use in older adults: results of a US consensus panel of experts. Arch Intern Med. Am Med Assoc 2003;163:2716.

9. Society AG, Society AG. American Geriatrics Society 2012 Beers Criteria Update Expert Panel. American Geriatrics Society updated Beers Criteria for potentially inappropriate medication use in older adults. J Am Geriatr Soc 20122012;60:616-31.

10. Society AG, Society AG. By the American Geriatrics Society 2015 Beers Criteria Update Expert Panel. American Geriatrics Society 2015 Updated Beers Criteria for Potentially Inappropriate Medication Use in Older Adults. J Am Geriatr Soc 20152015;63:2227-46.

11. Bradley MC, Fahey T, Cahir C, et al. Potentially inappropriate prescribing and cost outcomes for older people: a cross-sectional study using the Northern Ireland Enhanced Prescribing Database. Eur J Clin Pharmacol 2012;68:1425-33.
12. Shah SM, Carey IM, Harris T, et al. Quality of prescribing in care homes and the community in England and Wales. Br J Gen Pract 2012;62:329-36.

13. Goltz L, Kullak-Ublick GA, Kirch W. Potentially inappropriate prescribing for elderly outpatients in Germany: a retrospective claims data analysis. Int J Clin Pharmacol Ther 2012;50:185-94.

14. Bjerre LM, Ramsay T, Cahir C, et al. Assessing potentially inappropriate prescribing (PIP) and predicting patient outcomes in Ontario's older population: a population-based cohort study applying subsets of the STOPP/START and Beers' criteria in large health administrative databases. BMJ Open 2015;5:(e010 156):e010146.

15. Bronskill S, Carter M, Costa A, et al. Aging in Ontario : An ICES Chartbook of Health Service Use by Older Adults [Internet]. 2010 https://www.ices.on.ca/Publications/Atlases-and-Reports/2010/ Aging-in-Ontario

16. Institute for Clinical Evaluative Sciences (ICES). Health Services Data 2011 http://www.ices.on.ca/webpage.cfm?site_id=1\&org_id=26\& morg_id=0\&gsec_id=5314\&item_id=5326

17. Institute for Clinical Evaluative Sciences. ICES Data Dictionary [Internet]. 2016 https://datadictionary.ices.on.ca/Applications/ DataDictionary/Default.aspx

18. Institute for Clinical Evaluative Sciences. Datasets available through Data \& Analytic Services [Internet]. https://datadictionary.ices.on.ca/ applications/datadictionary/Default.aspx?viewmode=DataServices (cited 2 Feb 2018)

19. Bjerre L, Halil R, Catley C, et al. Identifying potentially inappropriate prescribing in whole populations: Coding the STOPP/START and Beers criteria for use with large, routinely collected population datasets. Manuscr Prep 2017.

20. Rockhill B, Newman B, Weinberg C. Use and misuse of population attributable fractions. Am J Public Health 1998;88:15-19.

21. Levin M. The occurence of lung cancer in man. Acta Union Int Contra Cancrum 1953;9:531-41.

22. Wodchis W, Buchmeneva K, Nikitovic M, et al. Guidelines on personlevel costing using administrative databases in Ontario [Internet. Toronto, 2013. Report No: 1. http://www.hsprn.ca/uploads/files/ Guidelines_on_PersonLevel_Costing_May_2013.pdf

23. Statistics Canada. Consumer price indexes. 2017.

24. Hohl CM, Zed PJ, Brubacher JR, et al. Do emergency physicians attribute drug-related emergency department visits to medicationrelated problems? Ann Emerg Med 2010;55:493-502.

25. Hohl CM, Robitaille C, Lord V, et al. Emergency physician recognition of adverse drug-related events in elder patients presenting to an emergency department. Acad Emerg Med 2005;12:197-205.

26. Boult C, Dowd B, McCaffrey D, et al. Screening elders for risk of hospital admission. J Am Geriatr Soc 1993;41:811-7.

27. Anderson GF, Steinberg EP. Predicting hospital readmissions in the Medicare population. Inquiry 1985;22:251-8.

28. Weissman JS, Stern RS, Epstein AM. The impact of patient socioeconomic status and other social factors on readmission: a prospective study in four Massachusetts hospitals. Inquiry 1994;31:163-72.

29. Krumholz HM, Parent EM, Tu N, et al. Readmission after hospitalization for congestive heart failure among Medicare beneficiaries. Arch Intern Med 1997;157:99-104.

30. Mh c LG. Correlates of early hospital readmission or death in patients with congestive heart failure. Am J Cardiol 1997:1640-4.

31. Hajjar ER, Cafiero AC, Hanlon JT. Polypharmacy in elderly patients. Am J Geriatr Pharmacother. Elsevier 2007;5:345-51.

32. Bates DW, Miller EB, Cullen DJ, et al. Patient risk factors for adverse drug events in hospitalized patients. ADE Prevention Study Group. Arch Intern Med 1999;159(21):2553-60.

33. Carey IM, De Wilde S, Harris T, et al. What factors predict potentially inappropriate primary care prescribing in older people? Analysis of UK primary care patient record database. Drugs Aging 2008;25:693-706.

34. Evans RS, Lloyd JF, Stoddard GJ, et al. Risk factors for adverse drug events: a 10-year analysis. Ann Pharmacother 2005;39(7-8):1161-8.

35. Kanjanarat $P$, Winterstein AG, Johns TE, et al. Nature of preventable adverse drug events in hospitals: a literature review. Am J Health Syst Pharm 2003;60(17):1750-9.

36. Dhalla IA, Anderson GM, Mamdani MM, et al. Inappropriate prescribing before and after nursing home admission. J Am Geriatr Soc 2002;50(6):995-1000.

37. Phillips RS, Safran C, Cleary PD, et al. Predicting emergency readmissions for patients discharged from the medical service of a teaching hospital. J Gen Intern Med 1987;2:400-5.

38. Reed RL, Pearlman RA, Buchner DM. Risk factors for early unplanned hospital readmission in the elderly. $J$ Gen Intern Med 1991;6:223-8. 
39. Corrigan JM, Martin JB. Identification of factors associated with hospital readmission and development of a predictive model. Health Serv Res 1992;27:81-101.

40. Colledge NR, Ford MJ. The early hospital readmission of elderly people. Scott Med J 1994;39:51-2.

41. van Walraven $\mathrm{C}$, Mamdani M, Fang J, et al. Continuity of care and patient outcomes after hospital discharge. J Gen Intern Med 2004;19:624-31.

42. SAS Institute Inc. SAS 9.4 Functions and CALL Routines: Reference. Fifth Edition. NC: Cary, 2016.
43. Weaver CG, Ravani P, Oliver MJ, et al. Analyzing hospitalization data: potential limitations of Poisson regression. Nephrol Dial Transplant 2015;30:1244-9.

44. Litman J. Prescribe Smart Application. Ottawa, ON, 2016.

45. WHO Collaborating Centre for Drug Statistics Methodology. ATC/DDD Index 2018 [Internet]. $2018 \mathrm{https} / / / w w w . w h o c c . n o / a t c \_d d d \_i n d e x /$

46. Ryan C, O'Mahony D, O'Donovan DÓ, et al. A comparison of the application of STOPP/START to patients' drug lists with and without clinical information. Int J Clin Pharm 2013;35:230-5. 